\title{
Electron screening in molecular fusion reactions
}

\author{
T. D. Shoppa ${ }^{1}$, M. Jeng ${ }^{1}$, S. E. Koonin ${ }^{1}$, K. Langanke ${ }^{1}$, and R. Seki ${ }^{1,2}$ \\ 1 W. K. Kellogg Radiation Laboratory, 106-38 \\ California Institute of Technology \\ Pasadena, CA 91125 USA \\ ${ }^{2}$ Department of Physics and Astronomy \\ California State University \\ Northridge, CA 91330
}

(September 4, 2018)

\begin{abstract}
Recent laboratory experiments have measured fusion cross sections at centerof-mass energies low enough for the effects of atomic and molecular electrons to be important. To extract the cross section for bare nuclei from these data (as required for astrophysical applications), it is necessary to understand these screening effects. We study electron screening effects in the low-energy collisions of $Z=1$ nuclei with hydrogen molecules. Our model is based on a dynamical evolution of the electron wavefunctions within the TDHF scheme, while the motion of the nuclei is treated classically. We find that at the currently accessible energies the screening effects depend strongly on the molecular orientation. The screening is found to be larger for molecular targets than for atomic targets, due to the reflection symmetry in the latter. The results agree fairly well with data measured for deuteron collisions on molecular deuterium and tritium targets.
\end{abstract}




\section{INTRODUCTION AND MOTIVATION}

The determination of nuclear cross sections at the astrophysically most effective energies requires in most cases an extrapolation of cross sections that have been measured at the lowest possible laboratory energies. It has been generally believed that the uncertainty in this extrapolation is reduced by lowering the energies at which data can be taken in the laboratory. However, Assenbaum et al. [1] pointed out that this strategy might be problematic as, at very low energies, the experimental cross sections do not reflect the collisions of bare nuclei. Rather, they are larger due to screening by the electrons present in the target and projectile. This screening has to be accounted for in a determination of the nuclear cross section required for astrophysical applications.

Screening by the target electrons has been observed in several low-energy fusion experiments ( [2] and references therein). Conventionally, the effects of electron screening are expressed in terms of an enhancement factor

$$
f(E)=\sigma_{\exp }(E) / \sigma_{\text {bare }}(E)
$$

where $\sigma_{\text {bare }}$ is the cross section for bare nuclei at center-of-mass energy $E$, and $\sigma_{\exp }$ is the cross section measured in the presence of target and projectile electrons. The experimental analysis generally assumes that the electrons can be thought of as effectively lowering the Coulomb energy between the two colliding nuclei by a constant and energy-independent energy increment $U_{e}$, the screening energy. Furthermore it is assumed that the bare-nuclei cross section (usually derived by extrapolation of data at higher energies, which are little affected by screening) is known. Realizing that $U_{e}<<E$ at those energies currently accessibly in the laboratory, the enhancement factor can be written as [四]

$$
\begin{aligned}
f(E) & =\frac{\sigma_{\text {bare }}\left(E+U_{e}\right)}{\sigma_{\text {bare }}(E)}=\frac{S\left(E+U_{e}\right)}{S(E)} \frac{E}{E+U_{e}} \frac{\exp \left(-2 \pi \eta\left(E+U_{e}\right)\right)}{\exp (-2 \pi \eta(E))} \\
& \approx \exp \left\{\pi \eta(E) \frac{U_{e}}{E}\right\} .
\end{aligned}
$$

Here, as is conventional in nuclear astrophysics, we have written the cross section in terms of the astrophysical $S$-factor 


$$
\sigma(E)=\frac{S(E)}{E} \exp (-2 \pi \eta(E)),
$$

where $\eta(E)=Z_{1} Z_{2} \alpha\left(\mu c^{2} / 2 E\right)^{\frac{1}{2}}$ is the Sommerfeld parameter for nuclei of charges $Z_{1}, Z_{2}$ and reduced mass $\mu$. As is obvious from the exponential energy dependence, electron screening effects rapidly become more important with decreasing collision energy.

The picture underlying the experimental analysis of electron screening effects has been confirmed in recent theoretical studies for atomic targets [3,[4]. In fact, these calculations show that, at those energies at which screening effects have been observed for atomic targets, the effective screening potential is nearly constant at distances smaller than the classical turning point and can be replaced by a spatially constant screening energy. Furthermore it has been found that, to a good approximation, the electrons can be treated adiabatically at these low energies, thus allowing one to replace the screening energy by the gain in binding energy between the united atom and the asymptotically separated fragment atoms. However, it is as yet unexplained why the experimentally determined screening energies apparently exceed the adiabatic limits in some cases [2].

A recent series of impressive experiments [2, [7] has established that the screening energy depends on the form of the target (atomic, molecular, solid, etc.). In particular, it has been reported that, for the same nuclear reaction, the electron screening is generally smaller for molecular targets than for atomic targets. This has been attributed [0] to the fact that, in the case of molecular targets, the gain in electron binding is reduced by the energy spent to break the molecular bond and by the energy transferred to the spectator nucleus in the molecule. Although this dynamical picture qualitatively explains the observed difference between molecular and atomic targets, it has not yet been tested theoretically.

To study screening in molecular targets, we have performed calculations of collisions of $Z=1$ nuclei with hydrogenic molecules. In our model the wave functions of the two electrons are evolved dynamically within the time dependent Hartree-Fock (TDHF) scheme, while the motion of the nuclei is treated classically. That is, we assume that the colliding nuclei behave as classical particles beyond the classical turning point, subject to the forces 
they exert on each other and to the force exerted on them by the electrons and the spectator nucleus. However, the electrons are treated quantum mechanically in the TDHF scheme, where the wave functions are calculated in the time-dependent potential generated by the nuclei and the inner-electron repulsion. In this way, we determine the value of the screening potential $U(R)$ between the two colliding nuclei. Additionally we monitor the force exerted on the spectator nucleus by the other charges to test the dynamical picture underlying the previous analysis of electron screening effects for molecular targets.

Our paper is organized as follows. In Section 2, we briefly describe the TDHF method applied in our calculation, as well as the numerical methods involved. Our results are presented and discussed in Section 3.

\section{THE TDHF METHOD}

We consider the collision of a $Z=1$ nucleus with a $Z=1$ target nucleus bound in a hydrogenic molecule. The nuclei can be treated as point-like and the collision as head-on, since the nuclear interaction occurs at distances far smaller than atomic scales. Our choice of nuclear coordinates is defined in Fig. 1. Initially, the electron distribution is cylindrically symmetric about the axis defined by the two molecular nuclei (z-axis). This symmetry is broken as the projectile nucleus approaches. We characterize the trajectory of the projectile nucleus by the orientation angle $\theta$ between the initial projectile velocity and the molecular axis. In our notation a collision where the projectile passes very near to the spectator nucleus is described by $\theta \approx 180^{\circ}$.

With our choice of coordinates the Hamiltonian for the problem reads

$$
H=\sum_{i=1,2} \frac{p_{i}^{2}}{2 m}+\sum_{j=p, s, t} \frac{P_{j}^{2}}{2 M_{j}}+V_{p t}+V_{p s}+V_{s t}+V_{e n}+V_{e e}
$$

where $m$ is the electron mass and the $M_{j}$ are the masses of the projectile (p), target (t) and spectator (s) nuclei. $V_{p t}, V_{s t}$, and $V_{p s}$ denote the various Coulomb repulsions among the nuclei. The Coulomb interaction between electrons (labeled $i=1,2$ ) and the nuclei is 


$$
V_{e n}=-\sum_{i=1,2} \sum_{j=p, s, t} \frac{e^{2}}{\left|\mathbf{r}_{i}-\mathbf{R}_{j}\right|}
$$

and $V_{e e}=e^{2} /\left|\mathbf{r}_{1}-\mathbf{r}_{2}\right|$ is the interaction between the two electrons.

The Hamiltonian for the isolated hydrogen molecule is given by

$$
H=\sum_{i=1,2} \frac{p_{i}^{2}}{2 m}+\sum_{j=s, t} \frac{P_{j}^{2}}{2 M_{j}}+V_{s t}+V_{e e}-\sum_{i=1,2} \sum_{j=s, t} \frac{e^{2}}{\left|\mathbf{r}_{i}-\mathbf{R}_{j}\right|} .
$$

In the Hartree-Fock approximation to the ground state, the electronic wave function can be written as

$$
\Psi\left(\mathbf{r}_{1}, \mathbf{r}_{2}, t\right)=\psi\left(\mathbf{r}_{1}, t\right) \psi\left(\mathbf{r}_{2}, t\right) \frac{1}{\sqrt{2}}(\alpha(1) \beta(2)-\beta(1) \alpha(2))
$$

This wave function describes two electrons in a spin-singlet state ( $\alpha$ and $\beta$ are the oneelectron spin states). As our Hamiltonian is independent of the spin coordinates, the spin structure of the electron wave function remains unchanged during the collision.

The initial ground state of the molecule was constructed by evolving a trial wavefunction with a fixed nuclear separation $R$ in imaginary time for the molecular Hamiltonian $H_{\text {mol }}$ [8,9], which damps out the highest energy components of the wave function. The resulting HF ground state is the lowest eigenfunction of the (discretized) Hamiltonian. As long as the overlap between the ground state and the trial wavefunction is nonzero, the wave function converges to the Hartree-Fock ground state. The nuclear separation has been treated as a variational parameter. We find the energy minimum at $R=0.78 \AA$, where the converged energy is $-30.8 \mathrm{eV}$. Both values are in good agreement with experiment.

We evolve the spatial wave function $\psi$ by solving the TDHF equation

$$
i \frac{\partial \psi(\mathbf{r}, t)}{\partial t}=-\left(\Phi(\mathbf{r}, t)+\nabla^{2}\right) \psi(\mathbf{r}, t)
$$

with

$$
\Phi(\mathbf{r}, t)=\frac{e^{2}}{\left|\mathbf{r}-\mathbf{R}_{s}(t)\right|}+\frac{e^{2}}{\left|\mathbf{r}-\mathbf{R}_{t}(t)\right|}+\frac{e^{2}}{\left|\mathbf{r}-\mathbf{R}_{p}(t)\right|}-e^{2} \int d^{3} \mathbf{r}^{\prime} \frac{\left|\psi\left(\mathbf{r}^{\prime}, t\right)\right|^{2}}{\left|\mathbf{r}-\mathbf{r}^{\prime}\right|}
$$

The electron-electron potential has been calculated by solving the appropriate Poisson equation subject to a spherical harmonics expansion around the center of electron charge as the 
boundary condition. The TDHF equations have been solved on a three-dimensional grid using a second-order expansion in $\Delta t$. The uniform Cartesian grid (with separation $0.26 \AA$ ) had $(20,20,40)$ points in $(x, y, z)$ directions, and $\psi$ was assumed to vanish at the boundaries.

In accordance with our classical treatment of the nuclei, the time dependence of the nuclear position vectors $\mathbf{R}_{i}(t)$ is determined from Newton's law, where the force on each nucleus is the sum of the Coulomb force of the other nucleus and the force due to the electronic charge density.

\section{RESULTS AND DISCUSSION}

The electron response of the target depends on the velocity of the incoming projectile. We have studied collisions of a $Z=1$ nucleus with a hydrogenic molecule for various collision angles $\theta$ and for projectile velocities between $0.2 \alpha c$ and $10 \alpha c$, corresponding to center-ofmass bombarding energies $E$ in the $d+\mathrm{D}_{2}$ system between $E=1 \mathrm{keV}$ and $2.5 \mathrm{MeV}$. In our discussion below we will refer to the velocity dependence of the collision in terms of the equivalent center-of-mass collision energy $E$ for the $d+\mathrm{D}_{2}$ system; for application to other hydrogenic reactions of interest, such as $d+\mathrm{T}_{2}$, these energies must be scaled according to the reduced mass of the colliding nuclei. In the calculation the projectile was aimed directly

at the target nucleus in the molecule, with an initial separation of $6 \AA$. We then followed the collision until the projectile had approached the target to the classical turning point.

In Figs. 2-4 we show "movies" of collisions at $E=2.5 \mathrm{MeV}, 25 \mathrm{keV}$, and $1 \mathrm{keV}$ at two selected angles, $\theta=57^{\circ}$ and $170^{\circ}$. The time evolution of the electron wave functions is represented by contour plots of electron densities, $2|\psi(\mathbf{r}, t)|^{2}$, projected onto the scattering plane spanned by the three nuclei. The positions of the nuclei at the various times were indicated by dots. In all cases, the initial electron configuration is the ground state of the hydrogen molecule.

At $E=2.5 \mathrm{MeV}$, the colliding nuclei move much faster than the average electron velocity. In this sudden limit, the projectile falls through the potentials generated by the electrons 
and the spectator nucleus, adding the respective potential energies to the collision energy. As expected, the electron densities at the classical turning point are independent of angle (Fig. 5). In the sudden limit, the collision energies are so high that electron screening has no influence on the nuclear process.

In the adiabatic limit, the target and projectile approach each other with a velocity much less than the average electron velocity. The electrons are thus able to respond nearly instantaneously to the nuclear motion and occupy the energetically most favorable configuration during the collision. This situation is illustrated by the collision at $E=1 \mathrm{keV}$. Upon reaching the classical turning point, the electron configuration corresponds to the ground state of a system of charges, $Z=2$ and $Z=1$, at the appropriate molecular separation. Fig. 6 shows the electron configurations at the classical turning point for various collision angles. Close inspection shows that the electron configurations vary with angles, so that the electrons are not entirely adiabatic even at this low collision energy (see below).

At $E=25 \mathrm{keV}$ (corresponding to a projectile velocity of $\alpha c$ ) the nuclear and electronic motions are comparable and the electron configuration is strongly distorted as the projectile passes through. Furthermore, the electron response depends strongly on the collision angle, as can be seen in Fig. 7.

During the collision we have monitored the expectation value of the forces exerted on the three nuclei by the other particles

$$
\mathbf{F}_{p}(t)=\int d^{3} \mathbf{r}^{\prime} \frac{\rho\left(\mathbf{r}^{\prime}, t\right)\left[\left(\mathbf{R}_{p}(t)-\mathbf{r}^{\prime}\right)\right]}{\left|\mathbf{R}_{p}(t)-\mathbf{r}^{\prime}\right|^{3}}-\frac{e^{2}\left(\mathbf{R}_{p}(t)-\mathbf{R}_{t}(t)\right)}{\left|\mathbf{R}_{p}(t)-\mathbf{R}_{t}(t)\right|^{3}}-\frac{e^{2}\left(\mathbf{R}_{p}(t)-\mathbf{R}_{s}(t)\right)}{\left|\mathbf{R}_{p}(t)-\mathbf{R}_{t}(t)\right|^{3}},
$$

with similar definitions for $\mathbf{F}_{t}, \mathbf{F}_{s}$. The relative force between projectile and target, that is exerted by the electrons and the spectator nucleus, along the collision trajectory then is

$$
\mathbf{F}_{\mathrm{rel}}(t)=\left(\mathbf{F}_{p}(t)-\mathbf{F}_{t}(t)\right) \cdot \frac{\mathbf{R}_{p}(t)-\mathbf{R}_{t}(t)}{\left|\mathbf{R}_{p}(t)-\mathbf{R}_{t}(t)\right|}=\left(\mathbf{F}_{p}(t)-\mathbf{F}_{t}(t)\right) \cdot \frac{\mathbf{R}_{\mathrm{rel}}}{\left|\mathbf{R}_{\mathrm{rel}}\right|},
$$

Upon integration of $F_{\text {rel }}$ along the collision trajectory we have determined the molecular screening potential $U_{\text {mol }}$, induced by the electrons and the spectator nucleus,

$$
U_{\mathrm{mol}}=\int \mathbf{F}_{\mathrm{rel}} \cdot d \mathbf{R}_{\mathrm{rel}}
$$


As an example, Fig. 8 shows the relative force and the screening potential for the collision at $E=25 \mathrm{keV}$ and at various angles. Depending on the relative dominance of the electrons and the spectator nucleus, the relative force is either attractive or repulsive, leading to an oscillatory behavior as a function of target-projectile separation. At large angles, the projectile passes through the repulsive Coulomb field of the spectator nucleus on its collision trajectory. In these cases, the exerted relative force is repulsive at a few atomic units. As expected, the effect is strongest at very large angles. After passing the spectator nucleus, the electron cloud dominates; the relative force becomes attractive again. When projectile and target are very close, the relative force must vanish.

The molecular screening potential is attractive in most cases, enhancing the fusion of projectile and target. However, at large angles, the screening potential can also become repulsive when the projectile passes the spectator nucleus.

As in [4] we interpret the screening potential at the classical turning point $\left(R_{c l}\right)$ as the (negative) screening energy, i.e. $\left(U_{e}(E, \theta)=-U_{\text {mol }}\left(R_{c l}, E, \theta\right)\right)$, which represents the net effect of spectator nucleus and electrons on the nuclear fusion process; i.e., we assume that the nuclei fuse with an effective energy $E+U_{e}$. Fig. 9 shows the screening energy as a function of collision energy at various angles.

The screening energies show a remarkable dependence on the scattering angle. At forward angles, $U_{e}(E, \theta)$ increases monotonically with decreasing collision energy. However, at back angles the screening energy shows a minimum near $E=25-30 \mathrm{keV}$. At extreme back angles $\left(\theta \geq 160^{\circ}\right)$ the minimum value even becomes negative, resulting in "anti-screening" (or depletion) of the fusion cross section under these kinematical conditions.

At low energies, the screening potential is attractive at all angles, and for $E \leq 3 \mathrm{keV}$ the electronic motions becomes adiabatic and $U_{e}(\theta)$ becomes nearly energy independent. However, even at these low energies its value depends noticeably on the molecular orientation. The screening energy is largest for $\theta \approx 90^{\circ}$, while it becomes smallest at large angles. This angle-dependence of the screening energy can be understood by considering the effect on the colliding nuclei of the electron density between the spectator and the target. At $\theta=90^{\circ}$ 
this electron density induces an attractive force between projectile and target. This force becomes less attractive with increasing or decreasing $\theta$ as the force component induced in the $z$-direction acts like a tidal force, actually hindering the fusing particles.

In our discussion we have ignored the rotation of the target molecule during the collision. This assumption is valid, as the angle $\Delta \theta$ that the molecule (with rotational energy $E_{\text {rot }}$ ) would rotate in the time it takes for the projectile (with kinetic energy $E$ ) to pass through the molecule is small. In particular,

$$
\Delta \theta \sim \sqrt{\frac{E_{\mathrm{rot}}}{E}}
$$

is only 0.005 radians in the most extreme case discussed here, where $E$ is $1 \mathrm{keV}$ and $E_{\text {rot }}$ is $0.025 \mathrm{eV}$.

For each screening energy $U_{e}(E, \theta)$ one can determine a corresponding enhancement factor $f(E, \theta)$ using Eq. (2). For a comparison with experiment, the enhancement factor has to be averaged over angle:

$$
\bar{f}(E)=\frac{1}{2} \int_{-1}^{1} f(E, \theta) d \cos (\theta)
$$

We have performed this average by 5-point Gaussian quadrature. Again using relation (2), $\bar{f}(E)$ can be expressed in terms of an average screening energy, $\bar{U}_{e}(E)$, which is shown in Fig. 10. As the extreme backward angles have only little weight in the average, $\bar{U}_{e}$ does not have a minimum at $E=25-30 \mathrm{keV}$ as one might expect from $U_{e}(\theta)$, but is a monotonically decreasing function of energy which changes from the sudden to the (nearly) adiabatic regime between $30 \mathrm{keV}$ and $3 \mathrm{keV}$.

Fig. 10 also shows the screening energy calculated for a deuteron colliding with an atomic D-target. The results are taken from the TDHF calculation of Ref. [4]. We observe that the screening energy at low collision energies is significantly larger for the $\mathrm{D}_{2}$-target than for the atomic D-target. We will argue that this is exceptional. It is the result of the reflection symmetry of the $\mathrm{d}+\mathrm{D}$ system. Asymptotically (large separations) the $\mathrm{d}+\mathrm{D}$ wave function is a $50 \%$ mixture of the gerade (positive parity) and ungerade (negative parity) configurations 
[3]. As parity is conserved during the collision, the united atom configuration in the adiabatic limit is then $1 / 2\left(\mathrm{He}^{+}(1 \mathrm{~s})+\mathrm{He}^{+}(2 \mathrm{p})\right)$, corresponding to a gain in electronic binding energy of 1.5 Ry during the collision. As this symmetry does not hold for the collision with the molecular target, the gain is larger and in the adiabatic limit is approximately given by 3 Ry, corresponding to an electron configuration similar to the one of $\mathrm{He}^{+}(1 \mathrm{~s})$.

More interestingly, Fig. 10 shows that the screening energy is larger in the atomic case than in the molecular case for energies $E \geq 20 \mathrm{keV}$. Obviously this is due to the fact that upon angle-averaging the screening in the molecular case is depleted due to the "antiscreening" effect when the projectile has to pass the spectator nucleus along its collision trajectory. We believe that this result is the main difference between collisions with molecular and atomic targets and will hold more generally. One might assume that the collision with the molecular target at small $\theta$ roughly imitates the collision on an atomic target. We then expect that, in general for colliding systems of non-identical charges the screening energy for an atomic target is larger than for a molecular target (see Fig. 9), in agreement with the observation reported in [7]. We note again that the reflection symmetry in the atomic $d+D$ system invalidates our general argument.

In our calculations we have also examined the force on the spectator nucleus. As an example, we consider the collision at $E=25 \mathrm{keV}$ and at $\theta=170^{\circ}$. We then calculate a gain of kinetic energy of the spectator nucleus of $\Delta E_{\text {kin }}=|\Delta \mathbf{P}|^{2} /(4 M) \approx 0.1 \mathrm{eV}$ (in the lab frame), where $\Delta \mathbf{P}=\int \mathbf{F}_{s}(t) d t$ is the momentum transfer to the spectator. This gain in kinetic energy is so small that the spectator does not move before the nuclear fusion process happens. We have verified that this is the case for all conditions studied here. Thus, we do not support the assumption that the breaking of the molecular bond and the kinetic energy transfered to the spectator decreases the screening energy in a collision with a molecular target relative to an atomic target [5,7,10]. We also find that the assumption that the projectile moves on a straight line trajectory is well justified for all orientations appearing in the Gaussian quadrature of Eq. (12).

In Ref. [10] the $d(d, p)$ and $d(d, n)$ reactions have been studied down to $E=1.6 \mathrm{keV}$ 
using a molecular target. Assuming a constant screening energy, the value $U_{e}=25 \pm 5 \mathrm{eV}$ has been deduced from the data 10$]$ between $1.6 \mathrm{keV}$ and $\approx 15 \mathrm{keV}$. As can be seen in Fig. 10, our calculation does not support the assumption of a constant screening energy in this energy regime, but predicts that $\bar{U}_{e}$ changes from $\approx 20 \mathrm{eV}$ at $E=15 \mathrm{keV}$ to $\bar{U}_{e}=40 \mathrm{eV}$ at $E=2 \mathrm{keV}$. Interpreting the screening energy deduced in Ref. [10] as an averaged value for the energy interval studied, our calculation appears to be in reasonable agreement with data. Fig. 11 compares our prediction for the enhancement of the $d+D$ cross section with the data (using Eq. (2) and our calculated $\bar{U}_{e}(E)$ ), assuming the same parameterized form of the bare-nuclei S-factor [11] as in Ref. [10].

Brown et al. 12 have measured the low-energy $t(d, n) \alpha$ cross section with high precision using a $T_{2}$ target. In Ref. [13] it has been argued that the cross section at energies $E \leq 16$ $\mathrm{keV}$ is enhanced due to screening effects. With this assumption and parameterizing the data for $E=16-70 \mathrm{keV}$ (which are not influenced by electron screening), by a Breit-Wigner resonance formula, the authors of [13] were able to consistently describe the Los Alamos data [12] at all energies $E \leq 70 \mathrm{keV}$, a task which previously failed without incorporation of screening effects. In Fig. 12 we in fact demonstrate that the $t(d, n) \alpha$ data are well described by adding the presently calculated screening energies to the parameterized bare nuclear S-factor, as given in 13 .

In conclusion, we have studied the collisions of hydrogen nuclei on hydrogenic molecules in a model that evolves the electron distributions dynamically within the TDHF method. Our particular interest has been the screening effects of the electrons on the two fusing nuclei. We have investigated this effect by determining the molecular screening potential, induced by the electrons and the spectator nucleus, on the two colliding nuclei. As in a previous study of collisions on atomic targets [4], we defined the screening energy as the negative value of the molecular screening potential at the classical turning point and assumed that the screening energy is added to the collision energy of the fusing particle. We found that the screening energy depends strongly on the molecular orientation. It is generally smaller if the projectile had to pass near the spectator nucleus on its collision trajectory. For the 
present case of collisions of $Z=1$ nuclei with a hydrogenic molecule we found that the screening energy is larger for the molecular target than for the atomic target. This is caused by the reflection symmetry in the latter system and we do not expect it to hold in the general case. We are planning to investigate this conjecture in future studies of helium collisions on hydrogenic targets.

The work was supported in part by the National Science Foundation, Grant Nos. PHY9412818 and PHY94-20470, and the U.S. DOE at CSUN, Grant No. DE-FG03-87ER40347. 


\section{REFERENCES}

[1] H. J. Assenbaum, K. Langanke, and C. Rolfs, Z. Phys. A327 (1987) 461

[2] E. Somorjai and C. Rolfs, Nucl. Instrum. Meth. 99 (1995) 297

[3] L. Bracci, G. Fiorentini, and G. Mezzorani, Phys. Lett. A146 (1990) 128

[4] T.D. Shoppa, S.E. Koonin, K. Langanke and R. Seki, Phys. Rev. 48 (1993) 837

[5] S. Engstler et al., Phys. Lett. B202 (1988) 179

[6] C. Angulo et al., Z. Phys. A345 (1993) 231

[7] P. Prati et al., Z. Phys. A350 (1994) 171

[8] K. Kulander, K.R. Sandhya Devi and S.E. Koonin, Phys. Rev. A25 (1982) 2968

[9] S.E. Koonin Computational Physics (Benjamin-Cummings, Menlo Park, 1986)

[10] U. Greife, F. Gorris, M. Junker, C. Rolfs and D. Zahnow, Z. Phys. 351 (1995) 107

[11] H.S. Bosch and G.M. Hale, Nucl. Fusion 32 (1992) 611

[12] R.E. Brown, N. Jarmie and G.M. Hale, Phys. Rev. C35 (1987) 1999; N. Jarmie, R.E. Brown and R.A. Hardekopf, Phys. Rev. C29 (1984) 2031

[13] K. Langanke and C. Rolfs, Mod. Phys. Lett. A22 (1989) 2101 


\section{FIGURES}

FIG. 1. Coordinates used in our study. The $z$-axis is defined by the intra molecular separation and $\theta$ is the angle between $\hat{z}$ and the target-projectile separation.

FIG. 2. Time evolution of the electron density for the collision with a hydrogen nucleus on a hydrogenic molecule at a collision energy of $E=2.5 \mathrm{MeV}$ and at $\theta=57^{\circ}$ (left side) and $\theta=170^{\circ}$ (right side). The electron density is plotted as equally spaced contours. The positions of the nuclei are marked by dots.

FIG. 3. Similar to Fig. 2, but for $E=25 \mathrm{keV}$.

FIG. 4. Similar to Fig. 2, but for $E=1 \mathrm{keV}$.

FIG. 5. Angular dependence of the electron density when target and projectile are separated by a distance corresponding to the classical turning point. The collision energy is $E=2.5 \mathrm{MeV}$.

FIG. 6. Similar to Fig. 5, but for $E=25 \mathrm{keV}$.

FIG. 7. Similar to Fig. 5, but for $E=1 \mathrm{keV}$.

FIG. 8. Relative force (in $\mathrm{eV} / \AA$, dashed line) and molecular screening potential (in $\mathrm{eV}$, solid line) for a collision of $E=25 \mathrm{keV}$ and at selected angles.

FIG. 9. Screening energy as a function of collision energy and at various angles.

FIG. 10. Comparison of the angle-averaged molecular screening energy $\bar{U}_{e}(E)$ (solid line) with the atomic screening energy (dashed line, from Ref. [4]) as a function of collision energy. 
FIG. 11. Comparison of the experimental $d(d, p)^{3} \mathrm{H}$ S-factors 10 with the bare-nuclei S-factor from Ref. [11] (dashed line) and our estimate in which electron screening is added to the bare-nuclei S-factor using Eq. (2).

FIG. 12. Same as Fig. 11, but for the $t(d, n) \alpha$ reaction. The experimental data are from [12], while the bare-nuclei S-factor is adopted from 13. 


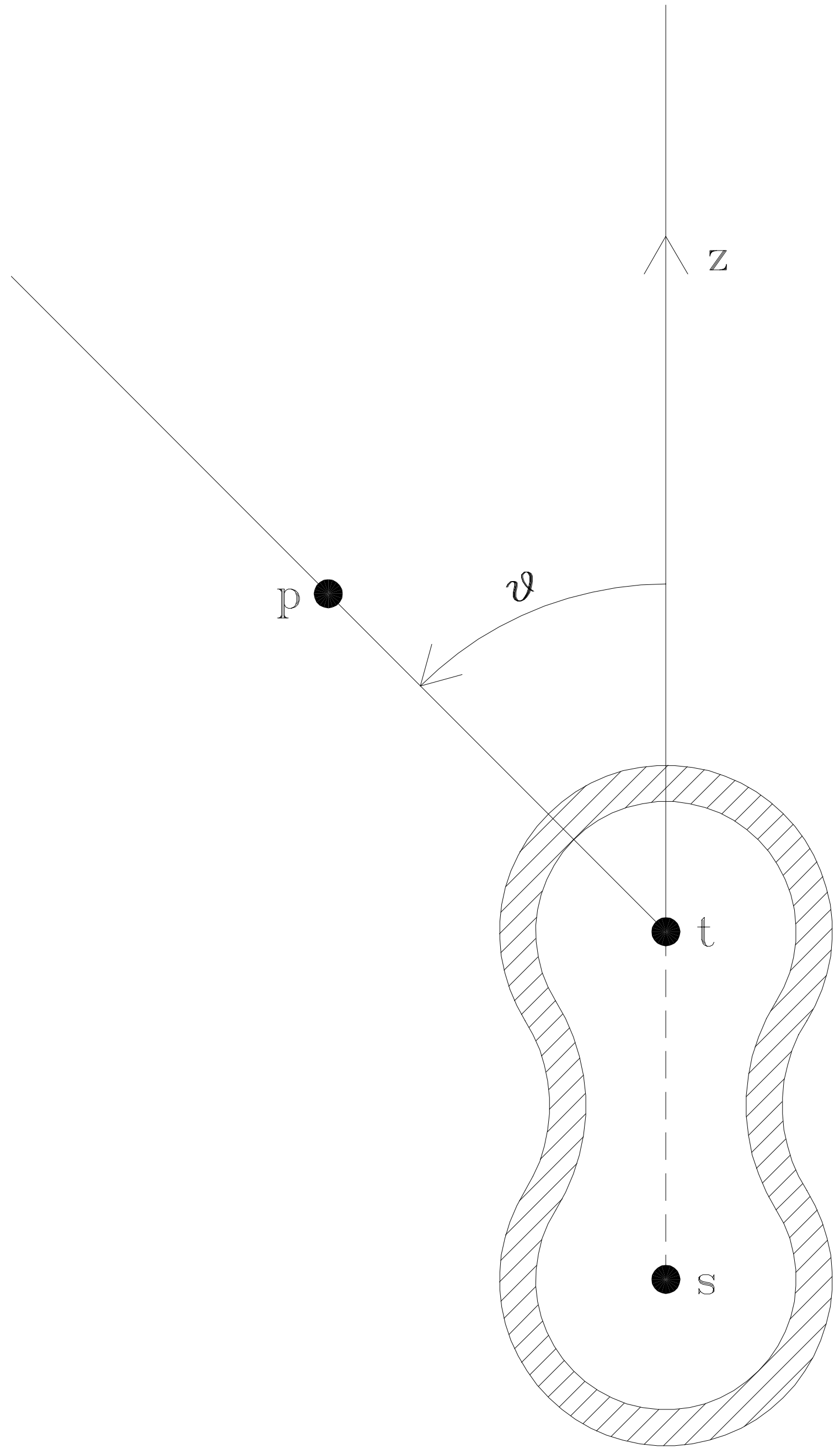



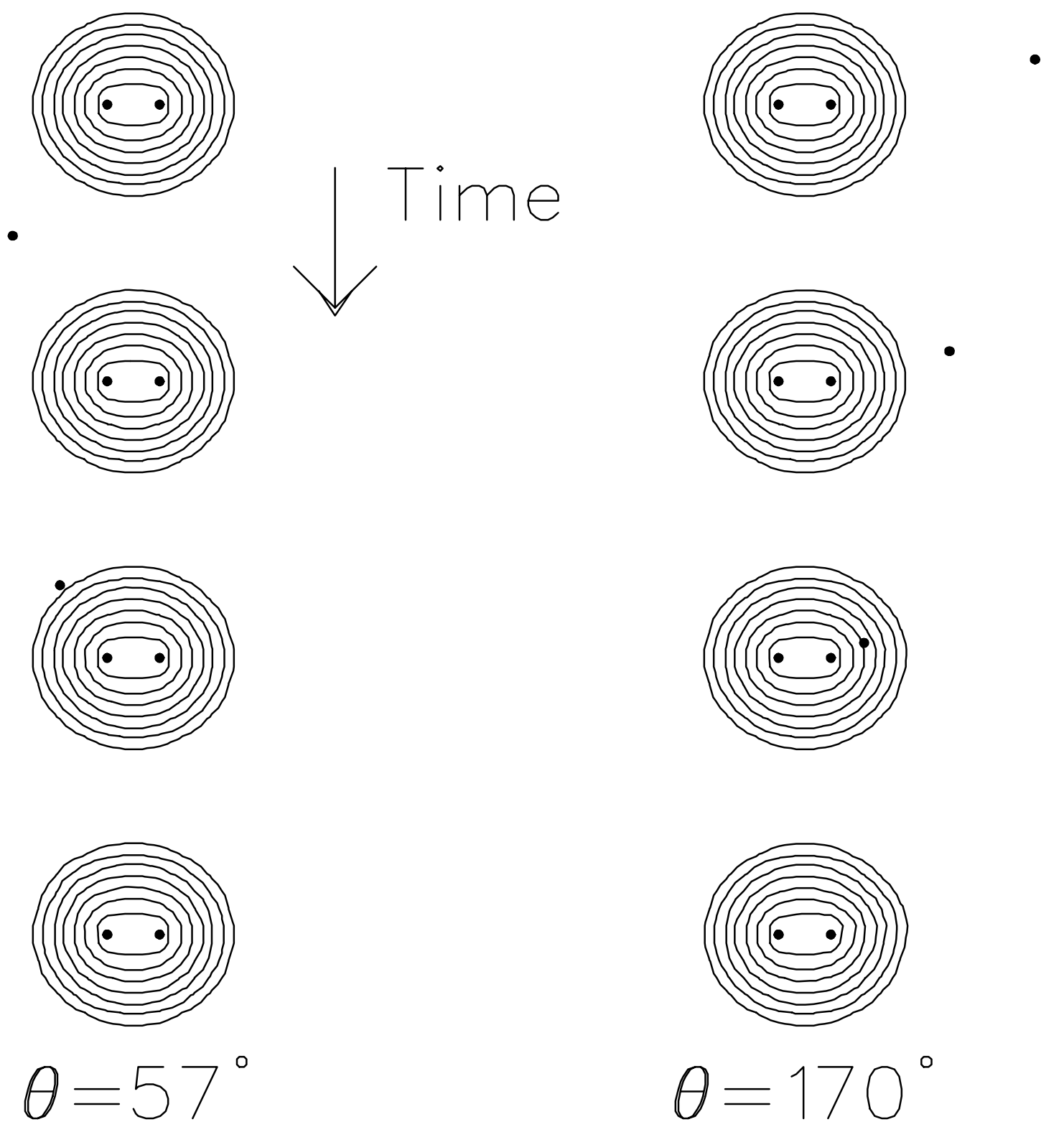

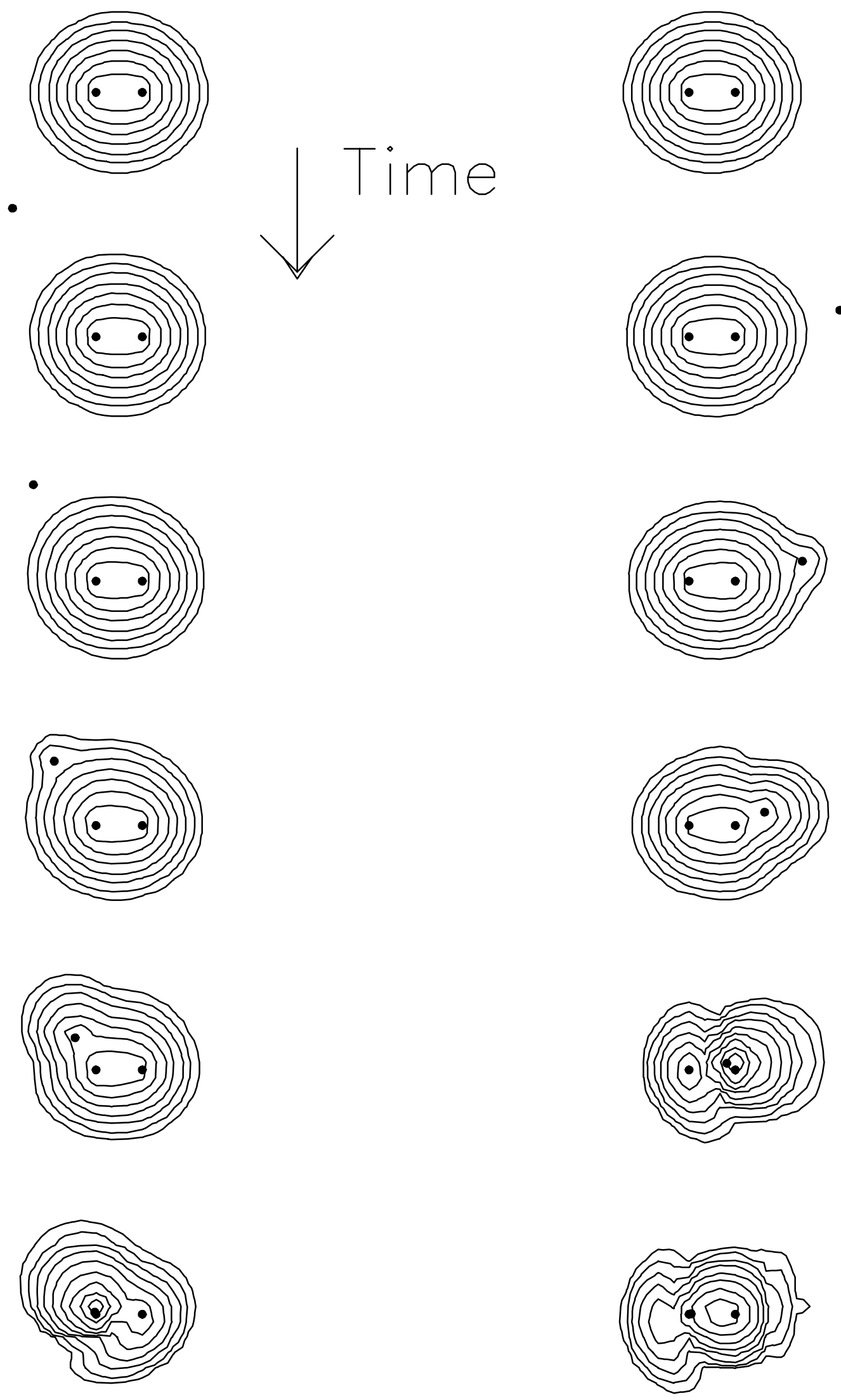

$\theta=57^{\circ}$

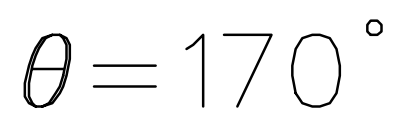



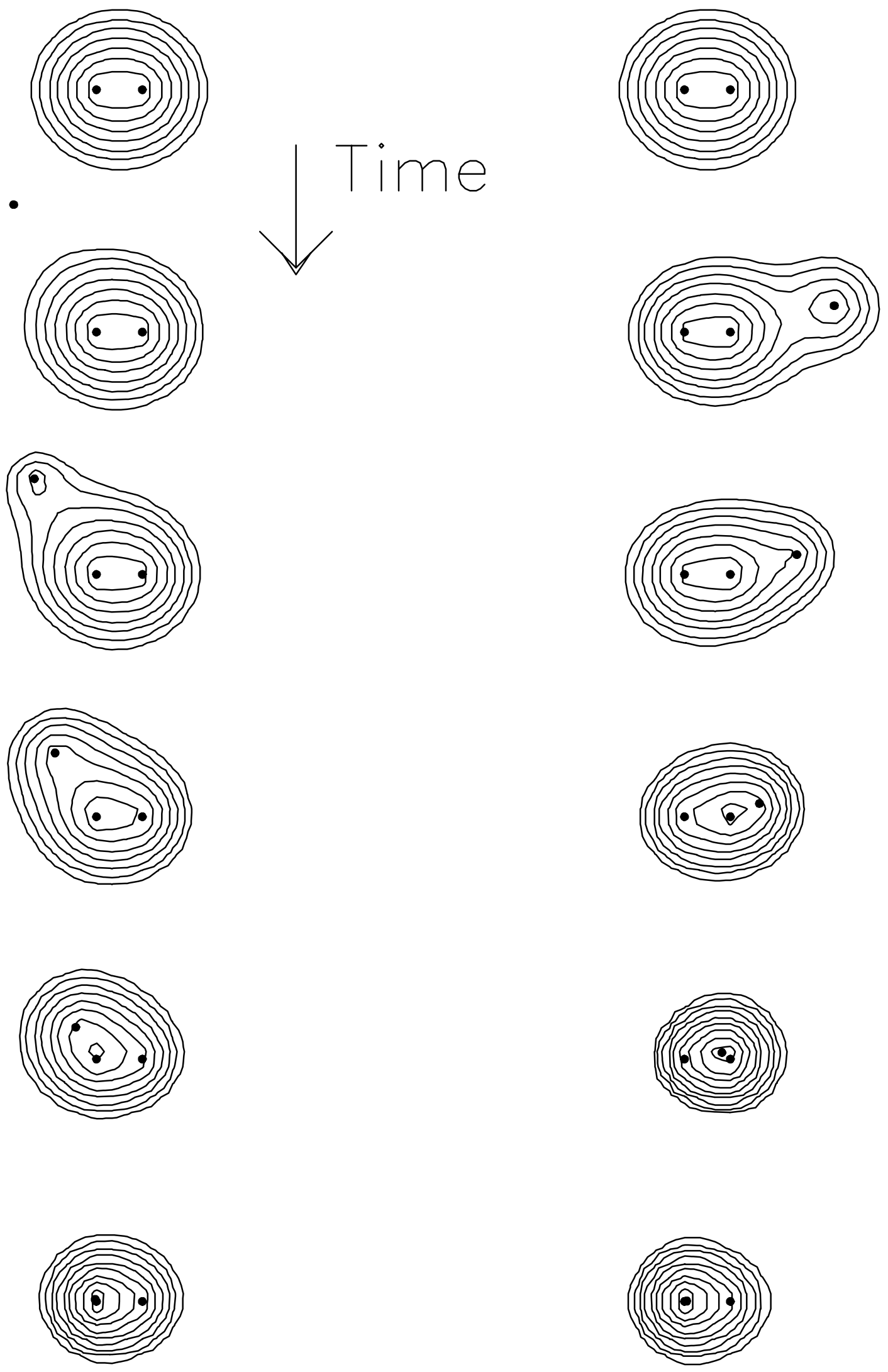

$\theta=57^{\circ}$

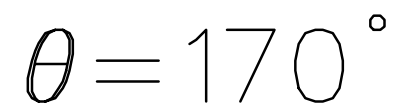



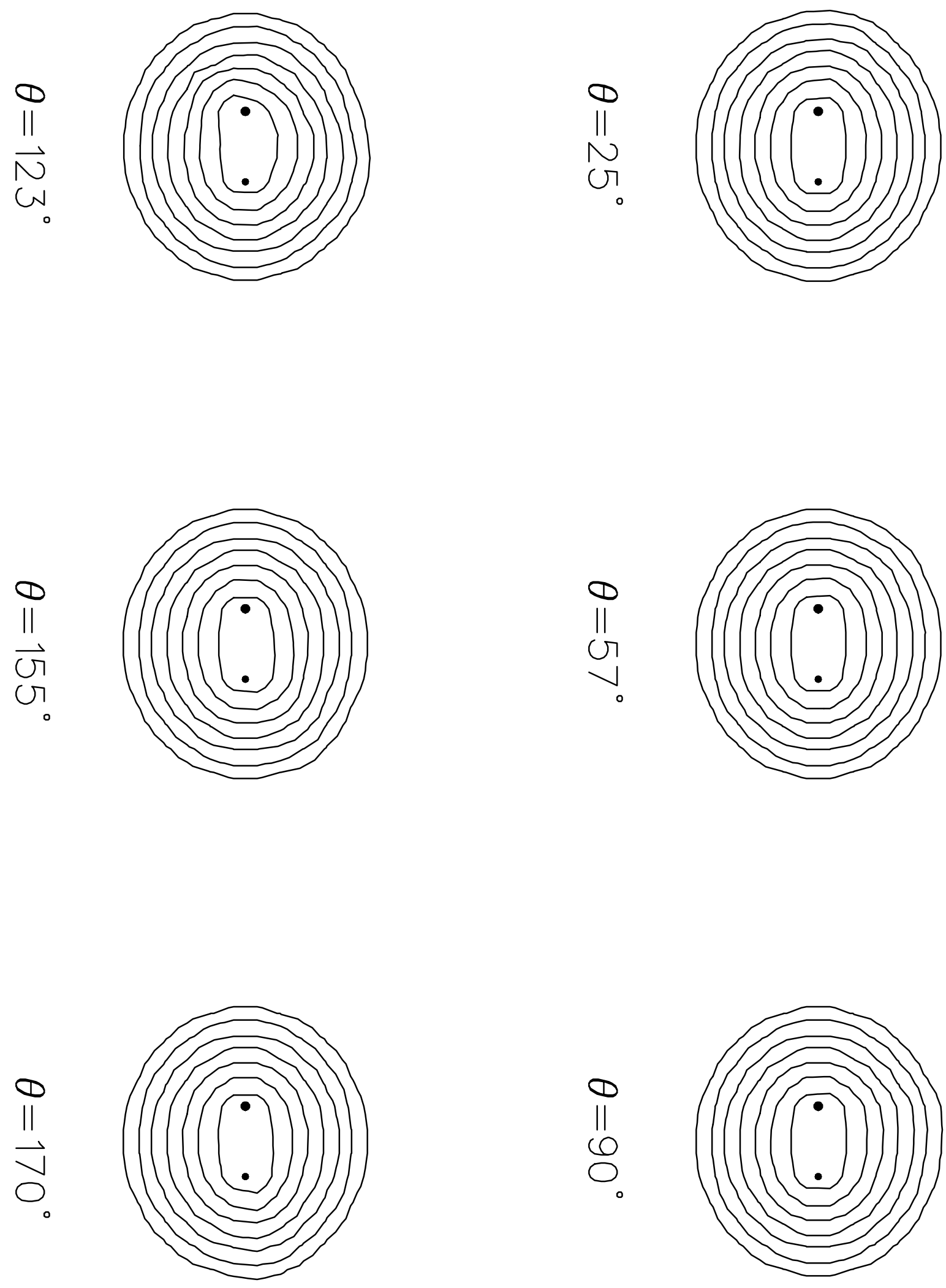

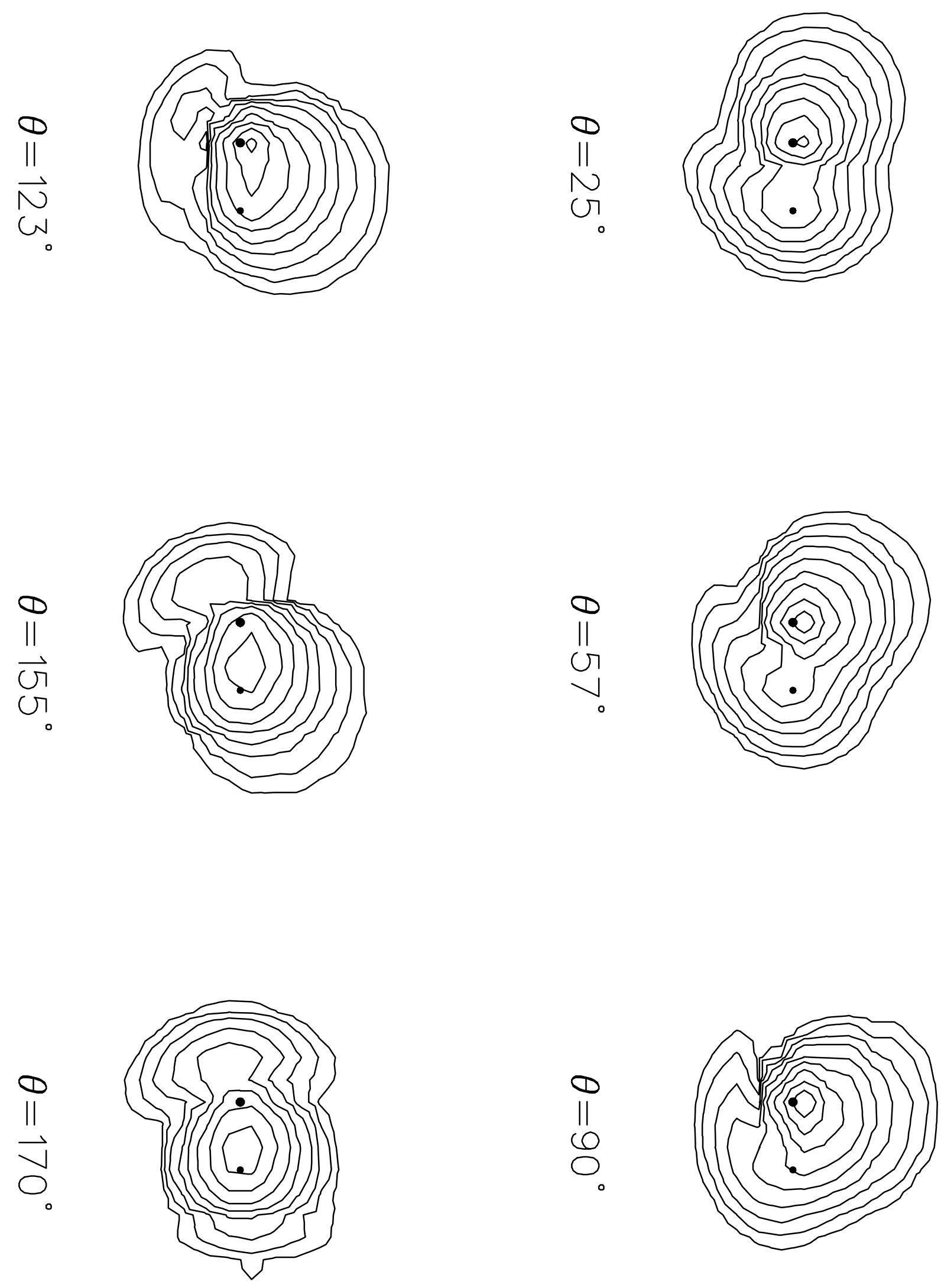

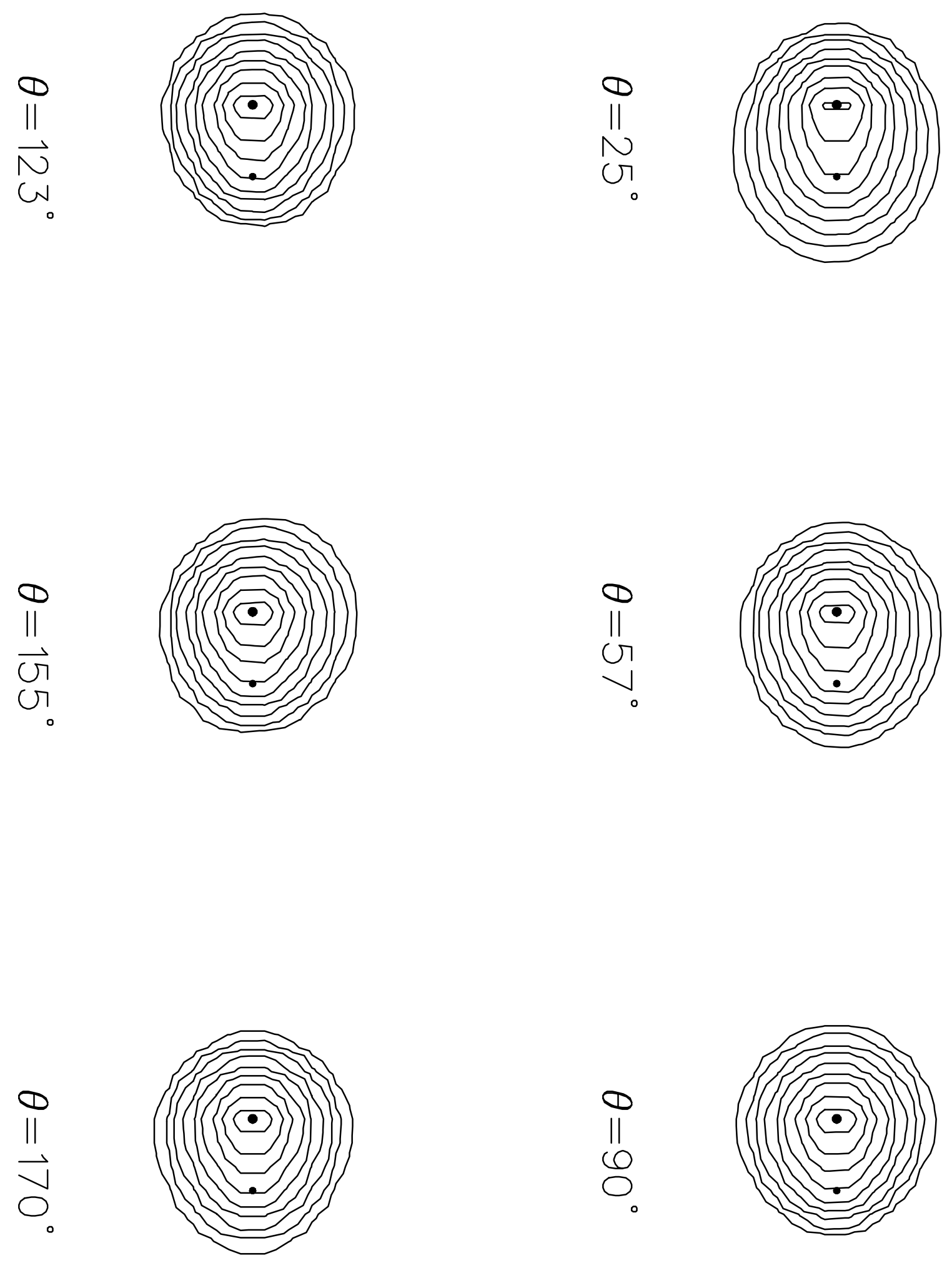

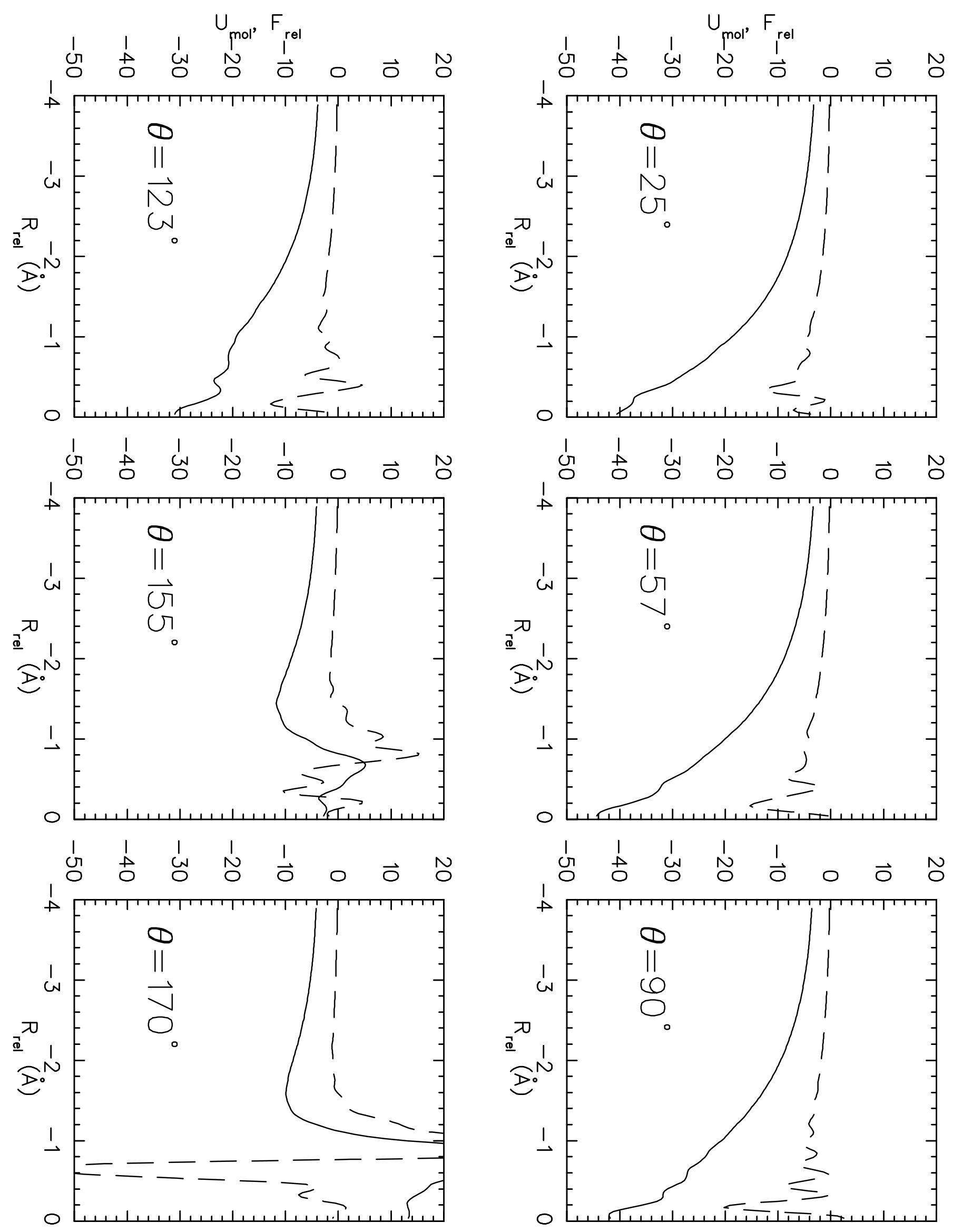


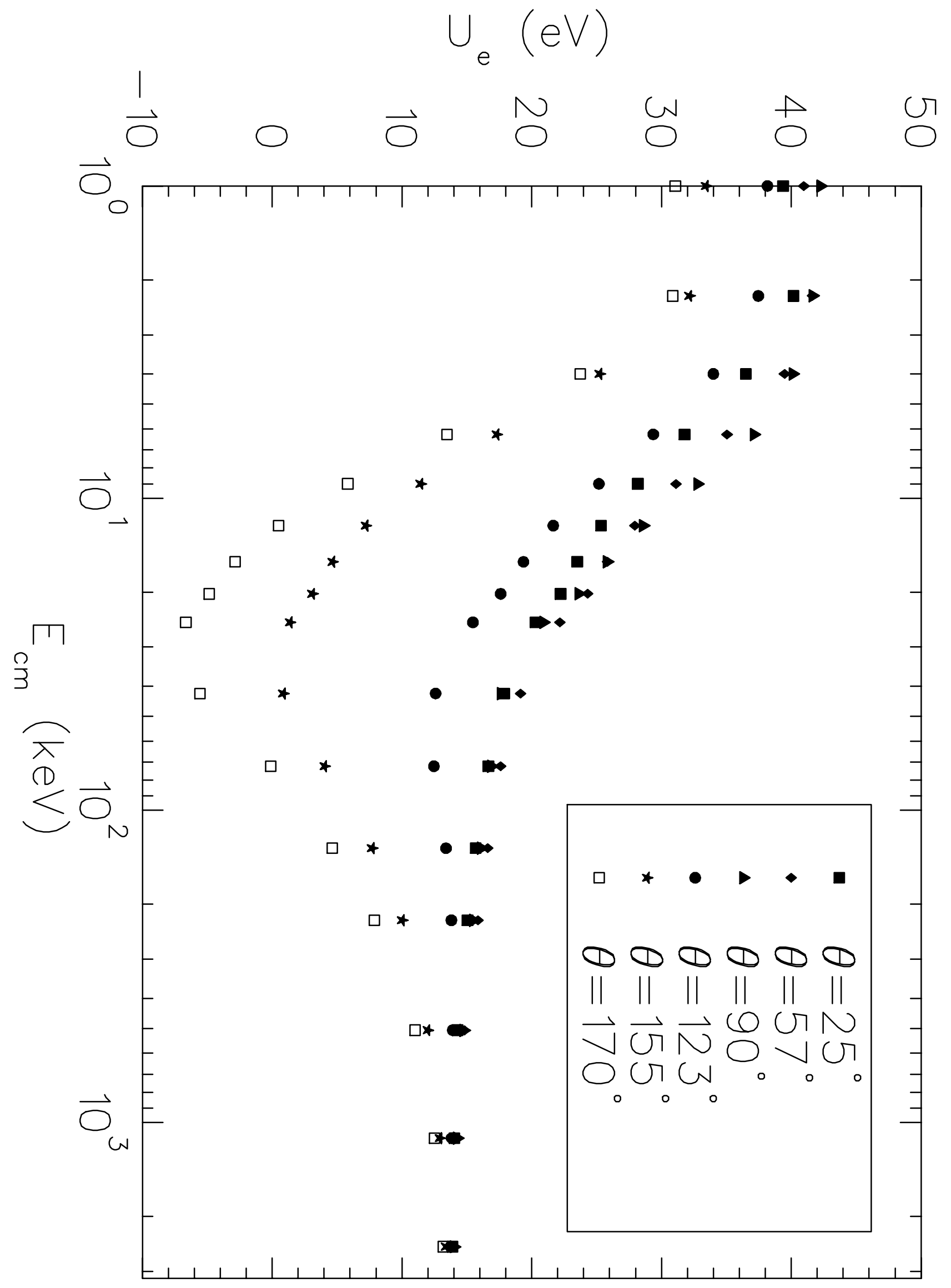




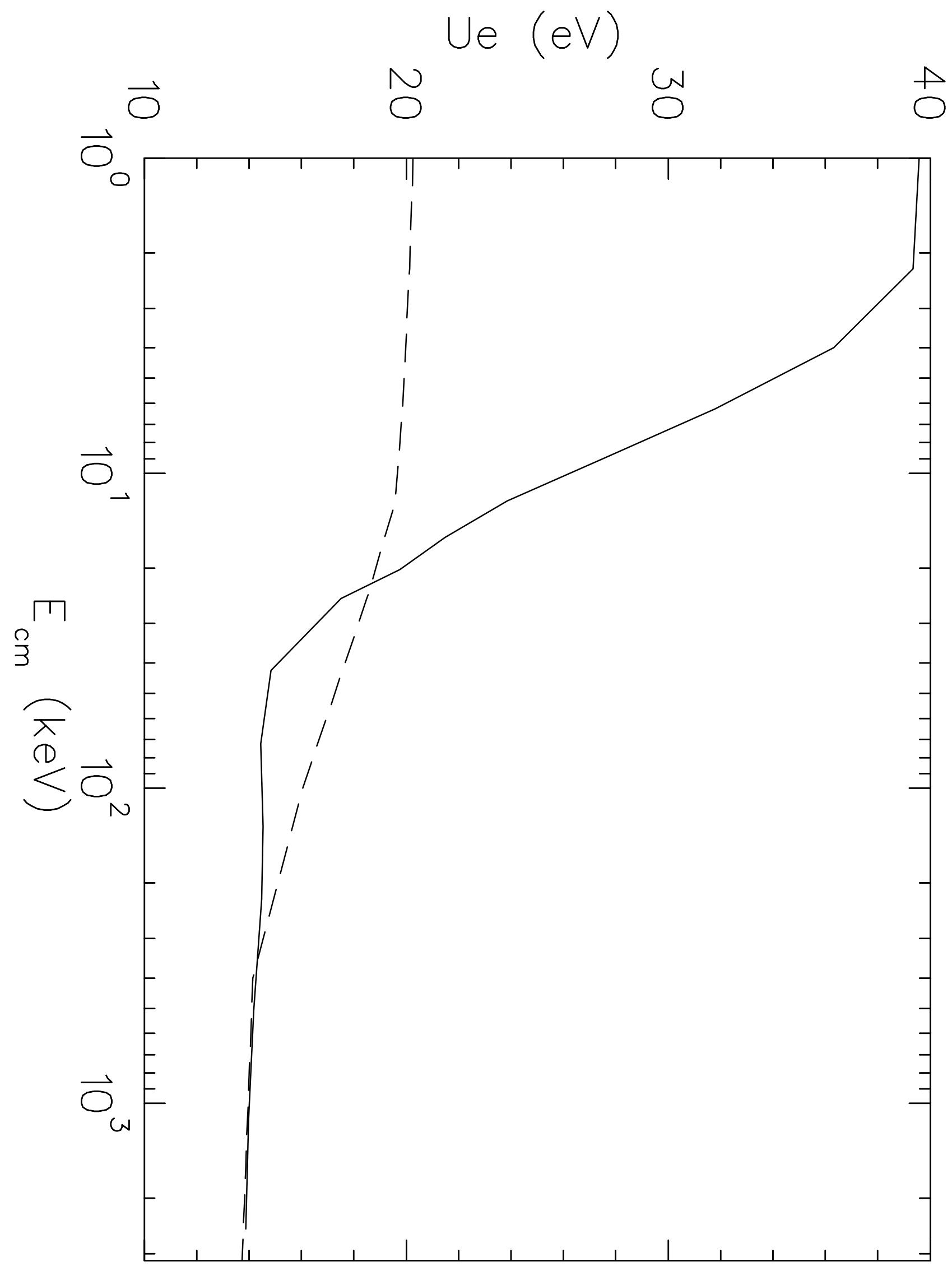




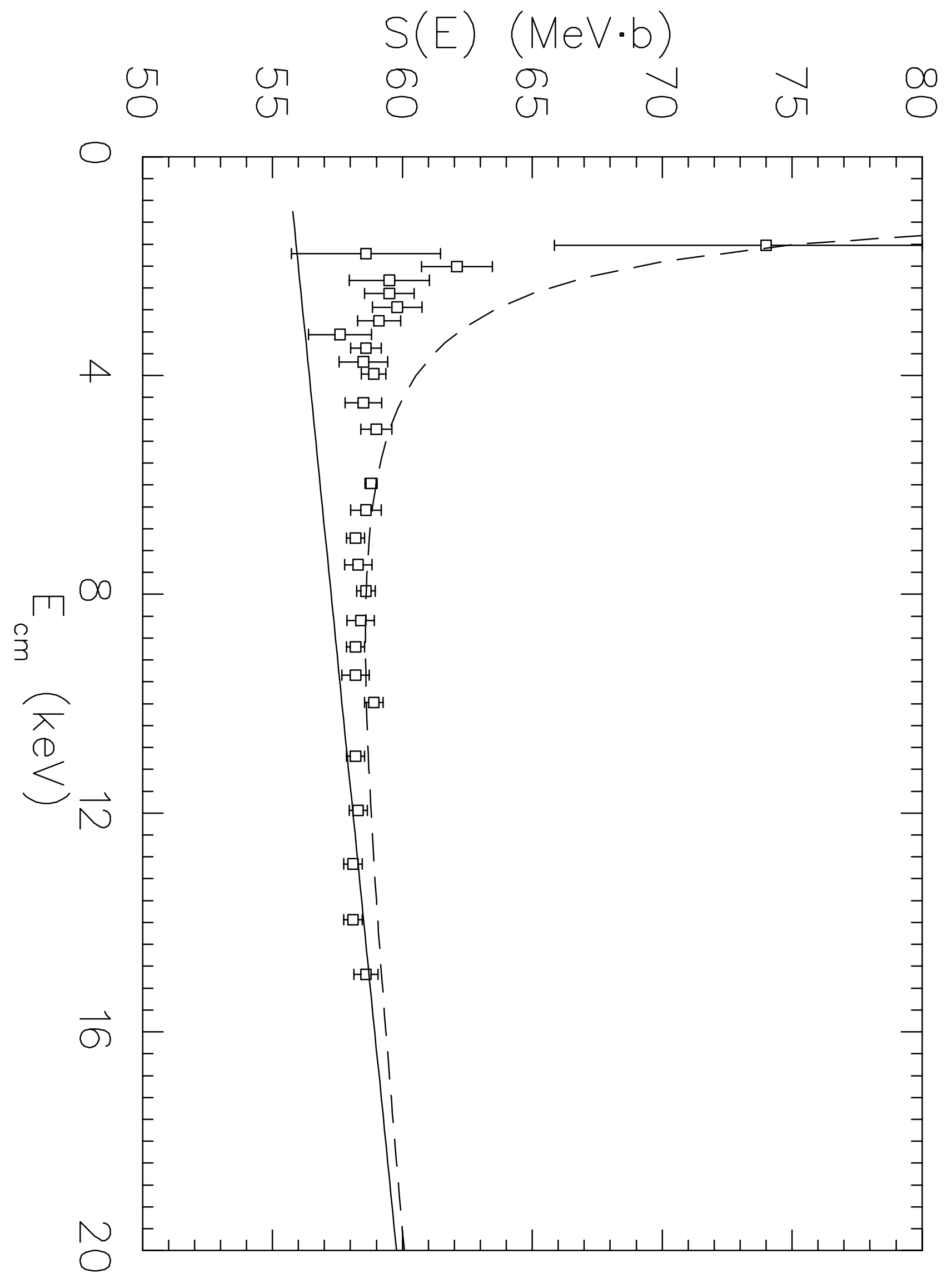




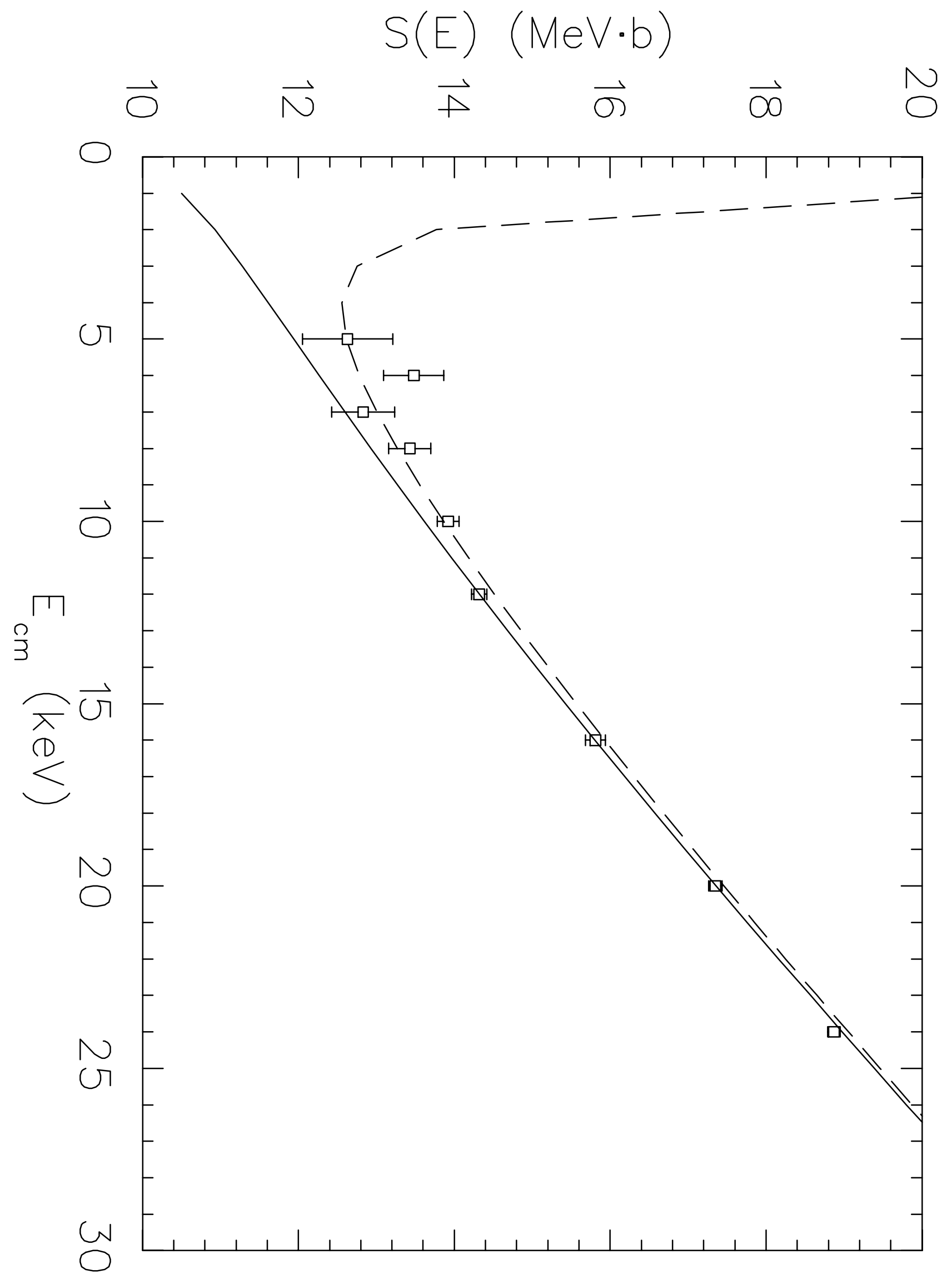

